\title{
NOTA
}

\section{ABSORÇÃO, TRANSLOCAÇÃO E UTILIZAÇÃO DE ZINCO, COBRE E MANGANÊS POR MUDAS ENXERTADAS DE Coffea arabica $^{(1)}$}

\author{
Marcelo Antonio Tomaz ${ }^{(2)}$, Herminia Emilia Pietro Martinez ${ }^{(3)}$, \\ Ney Sussumu Sakiyama ${ }^{(4)}$, Cosme Damião Cruz ${ }^{(5)} \&$ Antonio \\ Alves Pereira ${ }^{(6)}$
}

\begin{abstract}
RESUMO
Comparativamente ao pé-franco, a combinação enxerto/porta-enxerto altera os complexos mecanismos de "feedback" entre parte aérea e raízes, afetando de maneira positiva ou negativa a eficiência nutricional da planta. Este trabalho teve como objetivo avaliar, em cultivo hidropônico, a eficiência da absorção, translocação e utilização de Zn, Cu e Mn por mudas de Coffea arabica L., de acordo com o portaenxerto utilizado. $O$ experimento foi realizado em casa de vegetação, por um período de 170 dias, em vasos que continham areia como substrato, recebendo solução nutritiva circulante. Utilizaram-se, como enxerto, quatro genótipos de C. arabica: os cultivares Catuaí Vermelho IAC 15 e Oeiras MG 6851 e os híbridos 'H 419-10-3-15' e 'H 514-5-5-3', e, como porta-enxerto, quatro genótipos, sendo três de Coffea canephora Pierre ex Froenher: Apoatã LC 2258, Conilon Muriaé-1 e RC EMCAPA 8141 (recombinação entre clones da variedade Robustão CapixabaEMCAPA 8141) e uma linhagem de Coffea arabica L.: Mundo Novo IAC 376-4, além de quatro pés-francos. O delineamento experimental utilizado foi em blocos casualizados com 20 tratamentos, quatro repetições e uma planta por parcela. A eficiência nutricional das mudas quanto ao $\mathrm{Zn}, \mathrm{Cu}$ e $\mathrm{Mn}$ variou de acordo com a combinação enxerto/porta-enxerto. A progênie 'H 514-5-5-3' foi mais eficiente quanto à utilização de $\mathrm{Zn}$, Cu e Mn e produção de matéria seca, quando combinada com os porta-enxertos Apoatã LC 2258 e Mundo Novo IAC 376-4. O Catuaí Vermelho IAC 15 foi mais eficiente na utilização de $\mathrm{Cu}$ e Mn quando combinado com Apoatã LC 2258.
\end{abstract}

Termos de indexação: Coffea canephora, enxertia, hidroponia, solução nutritiva.

\footnotetext{
(1) Parte da Tese de doutorado do primeiro autor. Recebido para publicação em abril de 2004 e aprovado em fevereiro de 2006.

(2) Pós-Doutorando do Departamento de Fitotecnia, Universidade Federal de Viçosa - UFV. CEP 36571-000, Viçosa (MG). E-mail: tomazma@bol.com.br

(3) Professora do Departamento de Fitotecnia, UFV. E-mail: herminia@ufv.br

(4) Professor do Departamento de Fitotecnia, UFV. E-mail: sakiyama@ufv.br

${ }^{(5)}$ Professor do Departamento de Biologia Geral, UFV. E-mail: cdcruz@mail.ufv.br

(6) Pesquisador Empresa de Pesquisa Agropecuária de Minas Gerais - EPAMIG. CEP 36571-000 Viçosa (MG). E-mail: pereira@epamig.br
} 


\title{
SUMMARY: ABSORPTION, TRANSLOCATIONAND UTILIZATIONOF ZINC, COPPER AND MANGANESE IN GRAFTED SEEDLINGS OF Coffea arabica
}

\begin{abstract}
There are complexes feedback mechanisms involving the relationship rootstock/scion, whick can positively or negatively affect the plant nutrient efficiency use. The goal of the present work was to evaluate the efficiency of uptake, translocation and utilization of $Z n$, $\mathrm{Cu}$ and $\mathrm{Mn}$ in coffee seedlings. The experiment was carried out in a greenhouse over 170 days using circulating nutrient solution in pots with sand medium. Four Coffea arabica $L$. genotypes were used as grafts: Catuai Vermelho IAC 15 and Oeiras MG 6851, and the hybrids 'H 419-10-3-1-5' and 'H 514-5-5-3'. Three genotypes of Coffea canephora Pierre et Froenher were used as rootstocks: Apoatã LC 2258, Conilon Muriaé-1 and RC EMCAPA 8141 (recombination among clones of the variety Robustão Capixaba-EMCAPA 8141), one line of C. arabica: Mundo Novo IAC 376-4, and four additional non-grafted plants. The experiment was in a randomized block design with 20 treatments, four replications and one plant per plot. The nutritional efficiency of the coffee seedlings for $\mathrm{Zn}$, Cu and $\mathrm{Mn}$ varied according to the graft/rootstock combination. Progeny ' $H$ 514-5-5-3' proved to be the most effective regarding $\mathrm{Zn}$, $\mathrm{Cu}$ and $\mathrm{Mn}$ utilization and dry matter yield when combined with the rootstocks Apoatã LC 2258 and Mundo Novo IAC 376-4. Catuaí Vermelho IAC 15 was more effective for Cu and Mn utilization when combined with Apoatã LC 2258.
\end{abstract}

Index terms: Coffea canephora, grafting, hydroponics, nutritive solution.

\section{INTRODUÇÃO}

Muitas espécies e mesmo variedades de plantas diferem marcantemente em suas respostas à disponibilidade de nutrientes no solo (Anghinoni et al., 1989; Martinez et al., 1993; Fageria, 1998); as causas residem nas exigências nutricionais diferenciadas, na capacidade de absorção, de translocação (Li et al., 1991) e de utilização dos nutrientes (Siddiqi \& Glass 1981; Sands \& Mulligan 1990).

A busca de uma agricultura com menor consumo energético e ecologicamente sustentável tem estimulado a pesquisa a identificar mecanismos responsáveis pela maior eficiência nutricional, visando ao seu aproveitamento por meio de seleção e de outros métodos de melhoramento de plantas. Ademais, tem-se constatado ampla diversidade genética decorrente de uma série de mecanismos físiológicos, morfológicos e bioquímicos desenvolvidos pelas plantas quando submetidas às condições adversas de fertilidade do solo (Moura et al., 1999). Como a absorção, o transporte e a redistribuição de nutrientes apresentam controle genético, existe a possibilidade de melhorar e, ou, selecionar cultivares mais eficientes quanto à utilização de nutrientes (Gabelman \& Gerloff, 1983).

A enxertia do cafeeiro é uma prática, na qual se aproveita a qualidade do sistema radicular de determinados genótipos usados como portaenxertos, aliada às características de alta produtividade e qualidade de bebida do Coffea arabica L., usado como enxerto.
Trabalhos de enxertia do cafeeiro com o objetivo de estudar a absorção de micronutrientes são bastante escassos. Em culturas como fruteiras, nas quais o processo de enxertia já é estudado há mais tempo, trabalhos têm demonstrado a influência positiva da enxertia na absorção e na composição mineral, tanto de macro como de micronutrientes (Genú, 1985). Em estudos de absorção de macronutrientes por porta-enxertos de videira, em condições de hidroponia, Albuquerque \& Dechen (2000) observaram diferenças significativas nas concentrações de minerais de um mesmo cultivar enxertado sobre diferentes porta-enxertos.

Fahl et al. (1998), em experimento de enxertia de Coffea arabica L. sobre progênies de C. canephora e de $C$. congensis, verificaram que as plantas enxertadas apresentavam menores teores de Mn do que as não enxertadas.

Este trabalho teve como objetivo avaliar em cultivo hidropônico a eficiência de absorção, translocação e utilização de $\mathrm{Zn}, \mathrm{Cu}$ e $\mathrm{Mn}$ por mudas de C. arabica, de acordo com o porta-enxerto utilizado.

\section{MATERIAL E MÉTODOS}

O experimento foi realizado em casa de vegetação do Departamento de Fitotecnia da Universidade Federal de Viçosa, Minas Gerais, utilizando o cultivo em areia, fornecendo-se os nutrientes com solução nutritiva circulante. 
Utilizaram-se, como enxertos, quatro genótipos de C. arabica: os cultivares Catuaí Vermelho IAC 15 e Oeiras MG 6851 e as progênies 'H 419-10-3-1-5' e 'H 514-5-5-3', e, como porta-enxertos, quatro genótipos, sendo três de Coffea canephora Pierre ex Froenher: Apoatã LC 2258, Conilon Muriaé-1 e RC EMCAPA 8141 (recombinação entre clones da variedade Robustão Capixaba - EMCAPA 8141) e uma variedade de C. arabica: Mundo Novo IAC 376-4, além da utilização de quatro pés-francos (Quadro 1). A semeadura foi feita em caixas com areia lavada e peneirada. Estas caixas foram mantidas em casa de vegetação até que as plântulas atingissem o estádio "palito de fósforo", ocorrido 70 dias após o semeio. Depois deste período, efetuaram-se as enxertias que foram do tipo hipocotiledonar, conforme Moraes \& Franco (1973). Após a enxertia, as plantas foram transplantadas para caixas que continham areia peneirada lavada com água e mantidas em câmara de nebulização fechada por um período de 12 dias. Em seguida, foram colocadas em ambiente aberto, onde permaneceram por 15 dias para aclimatação, sendo irrigadas periodicamente. Depois de aclimatadas, as plantas foram levadas para casa de vegetação para a montagem do experimento.

O substrato utilizado foi areia peneirada, lavada e tratada com $\mathrm{HCl}$ concentrado comercial, para purificação do materiaL. A areia permaneceu no ácido por $24 \mathrm{~h}$, depois foi submetida a lavagens sucessivas com água corrente para retirar o excesso de ácido até que o $\mathrm{pH}$ atingisse valores em torno de 6,0 .

Foram utilizados vasos cilíndricos de plástico com capacidade de $3 \mathrm{~L}$. Esses foram perfurados no fundo, colocando-se uma mangueira de $1 / 2$ polegada para fazer a ligação com o recipiente coletor de solução nutritiva.

Empregou-se a solução de Clark (1975) modificada, com concentrações $\left(\mathrm{mmol} \mathrm{L}^{-1}\right)$ de 5,7 de $\mathrm{N}-\mathrm{NO}_{3} ; 1,0$ de $\mathrm{N}-\mathrm{NH}_{4}$; 0,1 de P; 2,4 de K; 1,2 de Ca; 0,6 de Mg; e 0,7 de S. Para os micronutrientes as concentrações $\left(\mu \mathrm{mol} \mathrm{L}^{-1}\right)$ foram de 19 de $\mathrm{B} ; 0,5$ de $\mathrm{Cu} ; 40$ de $\mathrm{Fe}$; 7,0de Mn; 0,09 de Mo; e 2,0 de Zn.

O delineamento experimental foi em blocos casualizados, com 20 tratamentos e quatro repetições, sendo quatro pés-francos e 16 combinações de enxertia (Quadro 1). Utilizou-se o teste " $t$ " de Student, a $5 \%$, para comparação entre as médias. O processamento foi realizado com o Aplicativo Computacional em Genética e Estatística - GENES (Cruz, 2001).

O transplantio para os vasos foi feito quatro meses após a semeadura, quando as plantas atingiram o estádio cotiledonar, fazendo-se a seleção quanto à uniformidade de tamanho e ao vigor da planta, colocando uma unidade por vaso. A parte superior da casa de vegetação foi recoberta com sombrite com malha de $50 \%$ para amenizar a insolação e a temperatura.

\section{Quadro 1. Relação das combinações de enxertia em mudas de genótipos de cafeeiro}

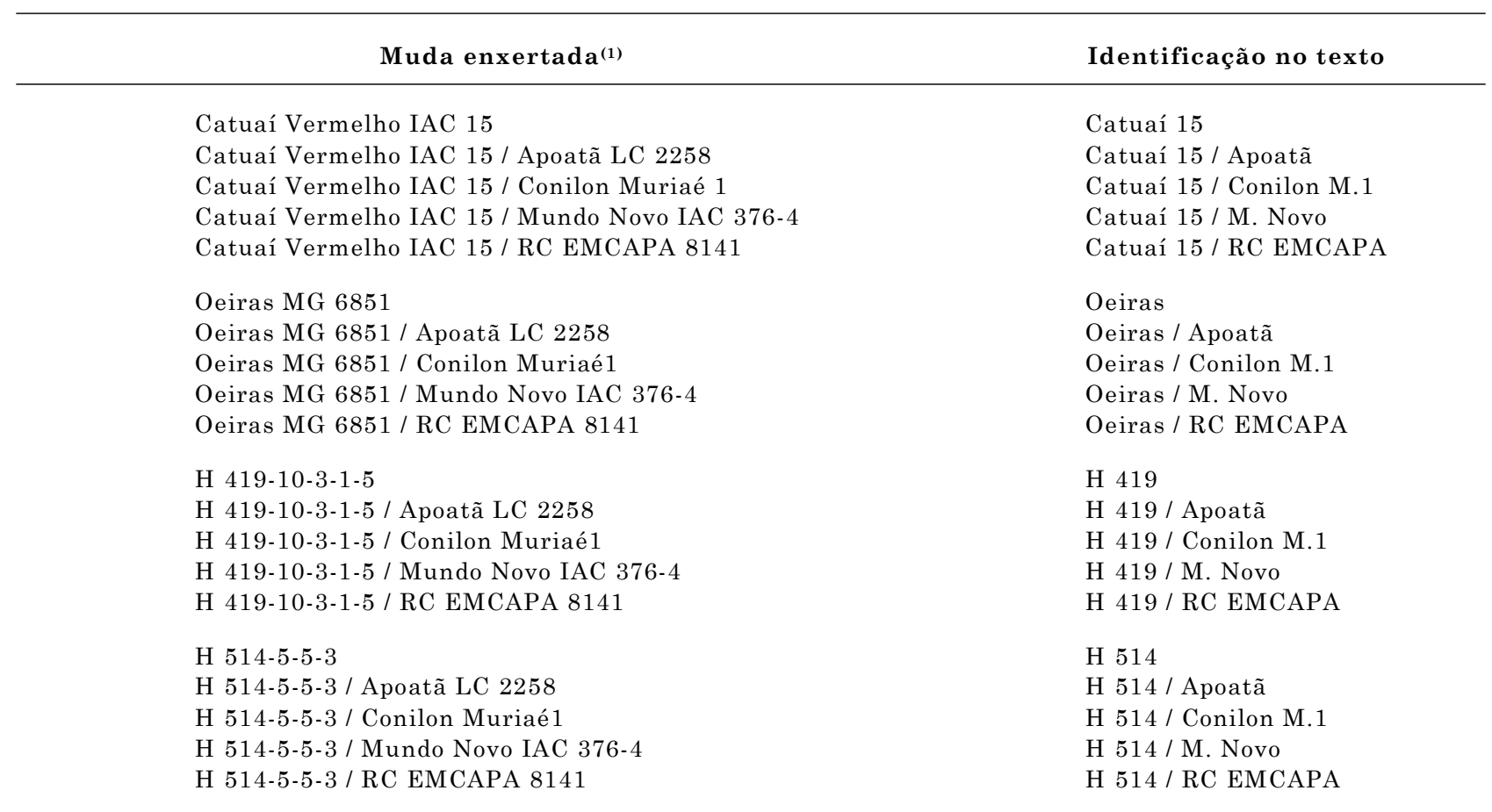

\footnotetext{
(1) Enxerto / porta-enxerto.
} 
Cada vaso recebeu 2,0 L de solução nutritiva e, à medida que ocorria diminuição do volume da solução em virtude da evapotranspiração, fez-se uma reposição com água deionizada até completar o volume de 2,0 L. A circulação da solução nutritiva foi realizada duas vezes ao dia. No decorrer do experimento, a concentração da solução foi aumentada mensalmente para 1,5 vez, 2,5 e 3,0 vezes, respectivamente, a da solução inicial. $\mathrm{O} \mathrm{pH}$ das soluções foi mantido a $5,5 \pm 0,5$ mediante ajustes diários com $\mathrm{NaOH}$. As trocas das soluções foram feitas periodicamente, quando a condutividade elétrica atingia $60 \% \pm 10 \%$ da CE inicial.

A avaliação foi realizada 170 dias após o transplantio para os vasos, separando-se a planta em raízes, caule e folhas, sendo todas as partes lavadas com água desmineralizada. Das raízes de cada tratamento, fez-se estimativa de comprimento radicular pelo método da intercepção de linha descrita por Tennant (1975).

Feitas as medições, os materiais foram secos em estufa com circulação forçada de ar a $70^{\circ} \mathrm{C}$, por $72 \mathrm{~h}$, pesados e triturados em moinho tipo Wiley. Os mesmos foram digeridos em mistura nítrico-perclórica (Johnson \& Ulrich, 1959), enquanto os teores de Zn, $\mathrm{Cu}$ e Mn foram determinados por espectrofotometria de absorção atômica (Malavolta et al., 1997). A partir da concentração dos nutrientes na planta e da matéria seca, foram calculados os índices: (a) eficiência de utilização de nutriente $=$ (matéria seca total produzida) ${ }^{2} /$ (conteúdo total do nutriente na planta); (b) eficiência de absorção = (conteúdo total do nutriente na planta / comprimento de raiz); (c) eficiência de translocação $=100 \times$ (conteúdo do nutriente na parte aérea / conteúdo total do nutriente na planta).

\section{RESULTADOS E DISCUSSÃO}

\section{Peso da matéria seca da parte aérea e total e comprimento de raiz}

A enxertia proporcionou aumentos da matéria seca da parte aérea (MSPA), e total (MST) das combinações H514/Apoatã e H514/M.Novo, mas acarretou decréscimos nas combinações Oeiras/ Apoatã, Oeiras/Conilon M.1, Oeiras/RC EMCAPA, H419/Conilon M.1, H419/RC EMCAPA, H514/ Conilon M.1 e H514/ RC EMCAPA (Quadro 2).

Comparando o sistema radicular dos pés-francos com as combinações de enxertias, verificou-se acréscimo significativo no comprimento das raízes nas combinações Catuaí 15/M.Novo, H514/M.Novo e redução nas combinações Catuaí 15/Conilon M.1, Oeiras/Apoatã, Oeiras/Conilon M.1, Oeiras/ M. Novo, Oeiras/RC EMCAPA , H 419/Conilon M.1, H419/RC EMCAPA, H514/Conilon M.1 e H514/ RC
Quadro 2. Matéria seca da parte aérea (MSPA), total (MST) e comprimento de raiz (LR) em materiais de café não enxertados (pé-franco) e enxertados em diversas combinações, em solução nutritiva e a significância dos contrastes

\begin{tabular}{|c|c|c|c|}
\hline Contraste & MSPA & MST & $\mathbf{L R}$ \\
\hline & \multicolumn{2}{|c|}{ - g planta pl $^{-}$} & $\mathrm{m}$ \\
\hline Catuaí 15 (pé-franco) & 3,69 & 4,75 & 29,55 \\
\hline vs Catuaí 15 / Apoatã & $4,45^{\mathrm{ns}}$ & $5,61^{\mathrm{ns}}$ & $33,04^{\mathrm{ns}}$ \\
\hline vs Catuaí 15 / Conilon M.1 & $2,66^{\mathrm{ns}}$ & $3,19^{\mathrm{ns}}$ & $20,01^{*}$ \\
\hline vs Catuaí 15 / M. Novo & $4,20^{\mathrm{ns}}$ & $5,51^{\mathrm{ns}}$ & $41,86^{*}$ \\
\hline vs Catuaí 15 / RC EMCAPA & $2,38^{\mathrm{ns}}$ & $3,28^{\mathrm{ns}}$ & $24,38^{\mathrm{ns}}$ \\
\hline Oeiras (pé-franco) & 5,59 & 7,32 & 52,14 \\
\hline vs Oeiras / Apoatã & $3,32^{*}$ & $4,34^{*}$ & $24,66^{*}$ \\
\hline vs Oeiras / Conilon M.1 & $2,06^{*}$ & $2,68^{*}$ & $14,64^{*}$ \\
\hline vs Oeiras / M. Novo & $5,29^{\mathrm{ns}}$ & $6,88^{\mathrm{ns}}$ & $41,40^{*}$ \\
\hline vs Oeiras / RC EMCAPA & $2,13^{*}$ & $2,67^{*}$ & $16,32^{*}$ \\
\hline H 419 (pé-franco) & 4,32 & 5,35 & 31,63 \\
\hline vs H 419 / Apoatã & $3,18^{\mathrm{ns}}$ & $4,27 \mathrm{~ns}$ & $32,98^{\mathrm{ns}}$ \\
\hline vs H 419 / Conilon M.1 & $1,71^{*}$ & $2,19^{*}$ & $11,84^{*}$ \\
\hline vs H 419 / M. Novo & $5,01^{\mathrm{ns}}$ & $6,46^{\mathrm{ns}}$ & $38,30^{\mathrm{ns}}$ \\
\hline vs H 419 / RC EMCAPA & $2,04^{*}$ & $2,59^{*}$ & $19,47^{*}$ \\
\hline H 514 (pé-franco) & 3,37 & 4,30 & 28,91 \\
\hline vs H 514 / Apoatã & $5,04^{*}$ & $6,43^{*}$ & $35,02^{\mathrm{ns}}$ \\
\hline vs H 514 / Conilon M.1 & $1,48^{*}$ & $1,85^{*}$ & $9,69^{*}$ \\
\hline vs H 514 / M. Novo & $5,60^{*}$ & $7,03^{*}$ & $42,28^{*}$ \\
\hline vs H 514 / RC EMCAPA & $2,08^{\mathrm{ns}}$ & $2,62^{*}$ & $17,95^{*}$ \\
\hline $\mathrm{CV}(\%)$ & 26,4 & 25,2 & 23,62 \\
\hline
\end{tabular}

* $\mathrm{e}^{\mathrm{ns}}$ : contrastes significativos e não-significativos, respectivamente, pelo teste "t" de Student a $5 \%$.

EMCAPA. É interessante observar que o maior comprimento do sistema radicular ocorreu em enxertia de genótipos de C. arabica em porta-enxerto da mesma espécie, o que pode indicar maior afinidade da copa e do porta-enxerto dentro da mesma espécie.

\section{Eficiência nutricional quanto ao zinco}

Observou-se aumento da eficiência de utilização de Zn (EUZn) de 70,5 e 64,2 \% respectivamente, para as combinações H514/Apoatã e H514/M.Novo, em relação ao pé-franco H514. No entanto, as combinações Catuaí 15/Conilon M.1, Oeiras/Conilon M.1, Oeiras/RC EMCAPA, H419/Conilon M.1, H419/ RC EMCAPA e H514/Conilon M.1 apresentaram reduções significativas quanto a (EUZn), em relação ao pé-franco, variando de 50,8 a 54,6 \% (Quadro 3).

Para Marschner, (1995), as diferenças genotípicas na eficiência nutricional podem estar relacionadas com a demanda de nutrientes em nível celular, compartimentalização, utilização na parte aérea, no 
Quadro 3. Eficiência quanto à utilização (EUZn), absorção (EAZn), translocação (ETZn) e conteúdo total de zinco (CTZn) em materiais de café não enxertados (pé-franco) e enxertados em diversas combinações, em solução nutritiva, e significância dos contrastes

\begin{tabular}{|c|c|c|c|c|}
\hline Contraste & EUZn & EAZn & ETZn & CTZn \\
\hline & $\mathrm{g}^{2} \mathrm{mg}^{-1}$ & $\mu \mathrm{g} \mathrm{m}^{-1}$ & $\%$ & $\mu \mathrm{g}$ planta $^{-1}$ \\
\hline Catuaí 15 (pé-franco) & 298,28 & 2,57 & 63,03 & 75,64 \\
\hline vs Catuaí 15 / Apoatã & $351,54^{\mathrm{ns}}$ & $2,71^{\mathrm{ns}}$ & $56,21^{\mathrm{ns}}$ & $89,38^{\mathrm{ns}}$ \\
\hline vs Catuaí 15 / Conilon M.1 & $141,40^{*}$ & $3,60^{*}$ & $61,65^{\mathrm{ns}}$ & $71,97^{\mathrm{ns}}$ \\
\hline vs Catuaí 15 / M. Novo & $324,87^{\mathrm{ns}}$ & $2,24^{\mathrm{ns}}$ & $64,26^{\mathrm{ns}}$ & $93,45^{\mathrm{ns}}$ \\
\hline vs Catuaí 15 / RC EMCAPA & $214,33^{\mathrm{ns}}$ & $2,06^{\mathrm{ns}}$ & $58,09^{\mathrm{ns}}$ & $50,19^{\mathrm{ns}}$ \\
\hline Oeiras (pé-franco) & 449,09 & 2,30 & 64,99 & 119,31 \\
\hline vs Oeiras / Apoatã & $331,03^{\mathrm{ns}}$ & $2,31^{\mathrm{ns}}$ & $63,08^{\mathrm{ns}}$ & $56,90^{*}$ \\
\hline vs Oeiras / Conilon M.1 & $203,96^{*}$ & $2,42^{\mathrm{ns}}$ & $59,91^{\mathrm{ns}}$ & $35,22^{*}$ \\
\hline vs Oeiras / M. Novo & $449,69^{\mathrm{ns}}$ & $2,56^{\mathrm{ns}}$ & $66,02^{\mathrm{ns}}$ & $105,26^{\mathrm{ns}}$ \\
\hline vs Oeiras / RC EMCAPA & $207,28^{*}$ & $2,11^{\mathrm{ns}}$ & $63,39^{\mathrm{ns}}$ & $34,40^{*}$ \\
\hline H 419 (pé-franco) & 355,43 & 2,56 & 61,23 & 80,53 \\
\hline vs H 419 / Apoatã & $327,22^{\mathrm{ns}}$ & $1,69^{*}$ & $56,86^{\mathrm{ns}}$ & $55,72^{\mathrm{ns}}$ \\
\hline vs H 419 / Conilon M.1 & $190,68^{*}$ & $2,13^{\mathrm{ns}}$ & $66,21^{\mathrm{ns}}$ & $25,15^{*}$ \\
\hline vs H 419 / M. Novo & $388,73^{\mathrm{ns}}$ & $2,83^{\mathrm{ns}}$ & $65,99^{\mathrm{ns}}$ & $107,35^{*}$ \\
\hline Vs H 419 / RC EMCAPA & $157,85^{*}$ & $2,18^{\mathrm{ns}}$ & $61,40^{\mathrm{ns}}$ & $42,50^{*}$ \\
\hline H 514 (pé-franco) & 269,52 & 2,38 & 57,40 & 68,60 \\
\hline vs H 514 / Apoatã & $459,46^{*}$ & $2,56^{\mathrm{ns}}$ & $60,07^{\mathrm{ns}}$ & $89,99^{\mathrm{ns}}$ \\
\hline vs H 514 / Conilon M.1 & $132,58^{*}$ & $2,67^{\mathrm{ns}}$ & $61,66^{\mathrm{ns}}$ & $25,82^{*}$ \\
\hline vs H 514 / M. Novo & $442,54^{*}$ & $2,65^{\mathrm{ns}}$ & $67,45^{*}$ & $111,68^{*}$ \\
\hline vs H 514 / RC EMCAPA & $174,75^{\mathrm{ns}}$ & $2,19^{\mathrm{ns}}$ & $56,68^{\mathrm{ns}}$ & $39,28^{*}$ \\
\hline $\mathrm{CV}(\%)$ & 28,6 & 18,8 & 11,2 & 27,1 \\
\hline
\end{tabular}

${ }^{*} \mathrm{e}^{\mathrm{ns}}$ : Contrastes significativos e não-significativos, respectivamente, pelo teste "t" de Student a $5 \%$.

transporte a curta e a longa distância, na afinidade do sistema de absorção (km), concentração mínima (Cmin) e modificações na rizosfera.

$\mathrm{O}$ aumento ou diminuição da utilização do Zn, nas combinações de enxertias estudadas, parece estar relacionado com a demanda diferenciada do nutriente no metabolismo da planta.

A análise dos resultados de conteúdo total de $\mathrm{Zn}$ (CTZn) na planta (Quadro 3) e da matéria seca total (MST) (Quadro 2) evidenciou que a taxa de aumento ou decréscimo dessas variáveis, na maioria das vezes, foi diretamente proporcional aos valores observados para EUZn.

Souza et al. (2001), avaliando o efeito de doses de $\mathrm{Zn}$ via solo em três cultivares de cafeeiro, observaram que a eficiência de utilização de $\mathrm{Zn}$ foi uma característica satisfatória para explicar a produção de matéria seca por cultivares de cafeeiro, na fase de muda, quando esse nutriente se encontrava em baixos teores no solo.

Aumento na eficiência de absorção de Zn (EAZn) ocorreu na combinação Catuaí 15/Conilon M.1 e diminuição no H419/Apoatã. Isto pode ter ocorrido pelo menor comprimento das raízes encontrado no Catuaí 15/Conilon M.1, que pode proporcionar um aumento da relação do nutriente absorvido por unidade de comprimento de raiz (Quadro 2) ou também pela baixa afinidade do sistema de absorção. No entanto, a redução da (EAZn), ocorrida na combinação H419/Apoatã, pode estar mais associada às características fisiológicas de absorção, visto que ela mesma não teve alteração significativa no comprimento de raiz, quando comparada com o respectivo pé-franco.

Analisando a eficiência de translocação de $\mathrm{Zn}$ (ETZn), verifica-se que a combinação H514/M.Novo apresentou aumento em relação ao pé-franco (Quadro 3). Esse acréscimo na translocação pode ter ocorrido pela maior concentração do nutriente na parte aérea em decorrência do aumento de produção de matéria seca, $66 \%$ a mais que o respectivo pé-franco (Quadro 2).

\section{Eficiência nutricional quanto ao cobre}

O contraste entre médias de mudas de pé-franco e enxertadas mostra que as combinações de enxertia Catuaí 15/Apoatã, H514/Apoatã e H514/M.Novo 
proporcionaram aumento de 52,5; 62,0; e 75,3\%, respectivamente, na eficiência quanto à utilização de $\mathrm{Cu}(\mathrm{EUCu})$ em relação aos respectivos pésfrancos. No entanto, os porta-enxertos Conilon M.1 e RC EMCAPA proporcionaram decréscimos significativos em todas as combinações de enxertias na (EUCu) (Quadro 4).

Aumento quanto à absorção de $\mathrm{Cu}(\mathrm{EACu})$ em $73 \%$ foi registrado na combinação Catuaí 15/Conilon M.1, quando comparado com o respectivo pé-franco. Para a eficiência de translocação de Cu (ETCu), ocorreram aumentos de 23,9; 19,8; 37,2 e 26,3\%, respectivamente, nas combinações Catuaí 15/ Conilon M.1, Oeiras/RC EMCAPA, H514/Conilon M.1 e H514/RC EMCAPA (Quadro 4).

A movimentação de íons através das raízes e seu descarregamento no xilema envolvem vários mecanismos que podem limitar sua ascensão para a parte aérea e podem constituir diferenças genotípicas na absorção e movimentação dos nutrientes (Gerloff \& Gabelman, 1983). A combinação Catuaí 15/Conilon M.1, apesar de ter sido eficiente quanto à absorção e à translocação de $\mathrm{Cu}$ (Quadro 4), não correspondeu na produção de matéria seca da planta (Quadro 2). Isto pode ter ocorrido pelo maior gasto de $\mathrm{Cu}$ no metabolismo e crescimento desta planta.

Quanto ao conteúdo total de $\mathrm{Cu}(\mathrm{CTCu})$ (Quadro 4) e MST (Quadro 2), os resultados foram semelhantes entre si. Os porta-enxertos Conilon M.1 e RC ENCAPA, quando combinados com Oeiras, H419 e H514, e mais a combinação Oeiras/Apoatã, tiveram resultados inferiores aos respectivos pésfrancos quanto a essas variáveis. Todavia, de todos os tratamentos, somente as combinações H514/ Apoatã e H514/M.Novo suplantaram os pés-francos quanto a estes índices.

Genú (1985), comparando o teor de Cu obtido em condição de pé-franco e enxertado em Citrus, verificou que determinados porta-enxertos levaram a acréscimos positivos de $\mathrm{Cu}$ nos enxertos, evidenciando a afinidade entre copa e porta-enxerto.

\section{Eficiência nutricional quanto ao manganês}

As combinações Catuaí15/Apoatã, H514/Apoatã, e H 514/M. Novo apresentram aumento significativo na eficiência de utilização de Mn (EUMn), quando comparadas com os respectivos pés-francos, da ordem de 59,9; 121,0 e $60,8 \%$, respectivamente. No entanto, houve redução de 44,7 a $57,4 \%$ da EUMn

Quadro 4. Eficiência quanto à utilização (EUCu), absorção (EACu), translocação (ETCu) e conteúdo total de cobre (CTCu) em materiais de café não enxertados (pé-franco) e enxertados em diversas combinações, em solução nutritiva, e significância dos contrastes

\begin{tabular}{|c|c|c|c|c|}
\hline Contraste & EUCu & EACu & ETCu & CTCu \\
\hline & $\mathrm{g}^{2} \mathrm{mg}^{-1}$ & $\mu \mathrm{g} \mathrm{m}^{-1}$ & $\%$ & $\mu \mathrm{g}$ planta $^{-1}$ \\
\hline Catuaí 15 (pé-franco) & 710,66 & 1,09 & 50,59 & 31,75 \\
\hline vs Catuaí 15 / Apoatã & $1.083,98^{*}$ & $0,89^{\mathrm{ns}}$ & $51,72^{\mathrm{ns}}$ & $29,03^{\mathrm{ns}}$ \\
\hline vs Catuaí 15 / Conilon M.1 & $276,47^{*}$ & $1,84^{*}$ & $62,70^{*}$ & $36,81^{\mathrm{ns}}$ \\
\hline vs Catuaí 15 / M. Novo & $781,15^{\mathrm{ns}}$ & $0,93^{\mathrm{ns}}$ & $55,25^{\mathrm{ns}}$ & $38,90^{\mathrm{ns}}$ \\
\hline vs Catuaí 15 / RC EMCAPA & $469,66^{*}$ & $0,94^{\mathrm{ns}}$ & $52,81^{\mathrm{ns}}$ & $22,91^{\mathrm{ns}}$ \\
\hline Oeiras (pé-franco) & 929,30 & 1,05 & 52,10 & 57,66 \\
\hline vs Oeiras / Apoatã & $840,02^{\mathrm{ns}}$ & $0,91^{\mathrm{ns}}$ & $47,23^{\mathrm{ns}}$ & $22,42^{*}$ \\
\hline vs Oeiras / Conilon M.1 & $466,75^{*}$ & $1,06^{\mathrm{ns}}$ & $54,83^{\mathrm{ns}}$ & $15,39^{*}$ \\
\hline vs Oeiras / M. Novo & $945,94^{\mathrm{ns}}$ & $1,21^{\mathrm{ns}}$ & $52,05^{\mathrm{ns}}$ & $50,04^{\mathrm{ns}}$ \\
\hline vs Oeiras / RC EMCAPA & $455,65^{*}$ & $0,96^{\mathrm{ns}}$ & $62,41^{*}$ & $15,65^{*}$ \\
\hline H 419 (pé-franco) & 952,49 & 0,96 & 53,16 & 30,05 \\
\hline vs H 419 / Apoatã & $766,40^{\mathrm{ns}}$ & $0,72^{\mathrm{ns}}$ & $45,57^{\mathrm{ns}}$ & $23,80^{\mathrm{ns}}$ \\
\hline vs H 419 / Conilon M.1 & $342,29^{*}$ & $1,17^{\mathrm{ns}}$ & $57,95^{\mathrm{ns}}$ & $14,01^{*}$ \\
\hline vs H 419 / M. Novo & $992,06^{\mathrm{ns}}$ & $1,10^{\mathrm{ns}}$ & $51,19^{\mathrm{ns}}$ & $42,07^{\mathrm{ns}}$ \\
\hline vs H 419 / RC EMCAPA & $367,83^{*}$ & $1,06^{\mathrm{ns}}$ & $61,42^{\mathrm{ns}}$ & $18,24^{*}$ \\
\hline H 514 (pé-franco) & 635,10 & 1,03 & 46,29 & 29,11 \\
\hline vs H 514 / Apoatã & $1.028,88^{*}$ & $1,15^{\mathrm{ns}}$ & $52,54^{\mathrm{ns}}$ & $40,18^{*}$ \\
\hline vs H 514 / Conilon M.1 & $281,22^{*}$ & $1,26^{\mathrm{ns}}$ & $63,52^{*}$ & $12,17^{*}$ \\
\hline vs H 514 / M. Novo & $1.113,29^{*}$ & $1,04^{\mathrm{ns}}$ & $54,30^{\mathrm{ns}}$ & $44,39^{*}$ \\
\hline vs H 514 / RC EMCAPA & $381,21^{*}$ & $1,01^{\mathrm{ns}}$ & $58,47^{*}$ & $18,01^{*}$ \\
\hline CV (\%) & 20,5 & 19,52 & 11,42 & 26,04 \\
\hline
\end{tabular}

“ ${ }^{\text {ns }}$ : Contrastes significativos e não-significativos, respectivamente, pelo teste "t” de Student a $5 \%$. 
Quadro 5. Eficiência quanto à utilização (EUMn), absorção (EAMn), translocação (ETMn) e conteúdo total de manganês (CTMn) em materiais de café não enxertados (pé-franco) e enxertados em diversas combinações, em solução nutritiva, e significância dos contrastes

\begin{tabular}{|c|c|c|c|c|}
\hline Contraste & EUMn & EAMn & ETMn & CTMn \\
\hline & $\mathrm{g}^{2} \mathrm{mg}^{-1}$ & $\mu \mathrm{g} \mathrm{m}^{-1}$ & $\%$ & $\mu \mathrm{g}$ planta ${ }^{-1}$ \\
\hline Catuaí 15 (pé-franco) & 18,95 & 41,53 & 88,60 & $1.190,63$ \\
\hline vs Catuaí 15 / Apoatã & $30,30^{*}$ & $31,52^{*}$ & $84,38^{\mathrm{ns}}$ & $1.038,68^{\mathrm{ns}}$ \\
\hline vs Catuaí 15 / Conilon M.1 & $15,21^{\mathrm{ns}}$ & $33,46^{\mathrm{ns}}$ & $88,91^{\mathrm{ns}}$ & $669,04^{*}$ \\
\hline vs Catuaí 15 / M. Novo & $22,54^{\mathrm{ns}}$ & $32,47^{\mathrm{ns}}$ & $89,02^{\mathrm{ns}}$ & $1.346,94^{\mathrm{ns}}$ \\
\hline vs Catuaí 15 / RC EMCAPA & $18,76^{\mathrm{ns}}$ & $23,01^{*}$ & $84,42^{\mathrm{ns}}$ & $573,48^{*}$ \\
\hline Oeiras (pé-franco) & 27,56 & 37,32 & 87,73 & $1.944,21$ \\
\hline vs Oeiras / Apoatã & $21,68^{\mathrm{ns}}$ & $35,26^{\mathrm{ns}}$ & $75,67^{*}$ & $868,80^{*}$ \\
\hline vs Oeiras / Conilon M.1 & $15,23^{*}$ & $32,22^{\mathrm{ns}}$ & $87,31^{\mathrm{ns}}$ & $471,60^{*}$ \\
\hline vs Oeiras / M. Novo & $27,91^{\mathrm{ns}}$ & $41,23^{\mathrm{ns}}$ & $87,72^{\mathrm{ns}}$ & $1.695,97^{\mathrm{ns}}$ \\
\hline vs Oeiras / RC EMCAPA & $15,07^{*}$ & $28,98^{\mathrm{ns}}$ & $88,78^{\mathrm{ns}}$ & $473,05^{*}$ \\
\hline H 419 (pé-franco) & 24,43 & 37,08 & 90,54 & $1.171,61$ \\
\hline vs H 419 / Apoatã & $25,74^{\mathrm{ns}}$ & $21,52^{*}$ & $81,6^{*}$ & $708,34^{*}$ \\
\hline vs H 419 / Conilon M.1 & $10,41^{*}$ & $38,92^{\mathrm{ns}}$ & $87,19^{\mathrm{ns}}$ & $460,72^{*}$ \\
\hline vs H 419 / M. Novo & $27,59^{\mathrm{ns}}$ & $40,02^{\mathrm{ns}}$ & $88,23^{\mathrm{ns}}$ & $1.512,56^{\mathrm{ns}}$ \\
\hline vs H 419 / RC EMCAPA & $11,44^{*}$ & $30,13^{\mathrm{ns}}$ & $87,13^{\mathrm{ns}}$ & $586,37^{*}$ \\
\hline H 514 (pé-franco) & 17,74 & 36,07 & 89,74 & $1.042,28$ \\
\hline vs H 514 / Apoatã & $39,22^{*}$ & $30,14^{\mathrm{ns}}$ & $81,81^{*}$ & $1.054,18^{\mathrm{ns}}$ \\
\hline vs H 514 / Conilon M.1 & $10,12^{\mathrm{ns}}$ & $35,01^{\mathrm{ns}}$ & $86,61^{\mathrm{ns}}$ & $338,19^{*}$ \\
\hline vs H 514 / M. Novo & $28,53^{*}$ & $40,94^{\mathrm{ns}}$ & $88,47 \mathrm{~ns}$ & $1.732,24^{*}$ \\
\hline vs H 514 / RC EMCAPA & $12,39^{\mathrm{ns}}$ & $30,87^{\mathrm{ns}}$ & $89,37^{\mathrm{ns}}$ & $554,03^{*}$ \\
\hline $\mathrm{CV}(\%)$ & 30,85 & 19,31 & 6,09 & 26,75 \\
\hline
\end{tabular}

" $\mathrm{e}^{\mathrm{ns}}$ : Contrastes significativos e não-significativos, respectivamente, pelo teste "t" de Student a $5 \%$.

para as combinações Oeiras/Conilon M.1, Oeiras/RC EMCAPA, H 419/Conilon M.1 e H 419/RC EMCAPA (Quadro 5).

Verificou-se que a combinação H514/M.Novo foi a única que mostrou aumento significativo do conteúdo total de $\mathrm{Mn}$ (CTMn), em 66,2\%. Entretanto, houve decréscimo do CTMn em todas as plantas enxertadas em Conilon e RC EMCAPA e mais as combinações Oeiras/Apoatã e H419/Apoatã, as quais variaram de 39,5 a 75,7 \%, respectivamente (Quadro 5).

Com relação à eficiência de absorção de $\mathrm{Mn}$ (EAMn), ocorreram reduções nas combinações Catuaí 15/Apoatã, Catuaí 15/RC EMCAPA e H 419/ Apoatã, quando comparadas aos respectivos pésfrancos. Quanto à eficiência de translocação de Mn (ETMn), verificou-se redução nas combinações com Apoatã, com exceção do Catuaí (Quadro 5).

Apesar de algumas combinações de enxertia apresentarem maior EUMn, estas não mostraram aumento na absorção e translocação do nutriente. Isto é importante em solos onde o Mn apresenta altos teores, uma vez que as plantas enxertadas poderiam ser menos alteradas por toxidez deste micronutriente.
Plantas da espécie de $C$. canephora são mais sensíveis ao Mn do que as de C. arabica (Willson, 1987). No entanto, a diminuição da absorção de Mn em plantas de $C$. canephora pode ter ocorrido por fatores ligados à seletividade de seu sistema radicular, não deixando que este micronutriente atinja concentrações prejudiciais à planta.

Fahl et al. (1998), avaliando enxertia de C. arabica sobre progênies de $C$. canephora e de C. congensis, observaram que, dentre os micronutrientes, houve decréscimo expressivo no teor de Mn nas folhas das plantas enxertadas, em relação às não enxertadas, tanto para o 'Catuaí' como para o 'Mundo Novo'.

Neste trabalho, de modo geral, as plantas enxertadas sobre materiais de Coffea canephora apresentaram também menores quantidades do micronutriente $\mathrm{Mn}$, dados esses que concordam com as afirmações de Fahl et al. (1998).

\section{CONCLUSÕES}

1. Houve variação da absorção, translocação e utilização de $\mathrm{Zn}$, Cu e Mn em mudas de cafeeiro, 
quando se compararam as combinações de enxertias com os respectivos pés-francos.

2. O cultivar Oeiras MG 6851 e a progênie 'H 41910-3-1-5', demonstraram ser eficientes como pésfrancos, não sendo beneficiados pelas enxertias testadas quanto à eficiência de absorção e utilização de $\mathrm{Zn}, \mathrm{Cu}$ e $\mathrm{Mn}$ e produção de matéria seca.

3. A progênie 'H 514-5-5-3', combinada com os porta-enxertos Apoatã e Mundo Novo, foi beneficiada na eficiência de utilização de $\mathrm{Zn}, \mathrm{Cu}, \mathrm{Mn}$ e produção de matéria seca.

4. Algumas combinações de enxertia apresentaram maior eficiência de utilização de $\mathrm{Mn}$, mas não apresentaram aumento na absorção e translocação deste nutriente.

\section{LITERATURA CITADA}

ALBUQUERQUE, T.C.S. \& DECHEN, A.R. Absorção de macronutrientes por porta-enxertos e cultivares de videira em hidroponia. Sci. Agríc., 57:135-139, 2000.

ANGHINONI, I.; VOLKART, C. R.; FATTORE, N. \& ERNANI, P.R. Morfologia de raízes e cinética da absorção de nutrientes em diversas espécies e genótipos de plantas. R. Bras. Ci. Solo, 13:355-361, 1989.

CLARK, R.B. Characterization of phosphatase of intact maize roots. J. Agric. Food Chem., 23:458-460, 1975.

CRUZ, C.D. Programa Genes: Aplicativo computacional em genética e estatística. Viçosa, Universidade Federal de Viçosa, 2001. 648p.

FAGERIA, N.K. Otimização da eficiência nutricional na produção das culturas. R. Bras. Eng. Agríc. Amb., 2:6-16, 1998.

FAHL, J.I.; CARELLI, M.L.C.; GALLO, P.B.; COSTA, W.M. \& NOVO, M.C.S.S. Enxertia de Coffea arabica sobre progênies de $C$. canephora e de $C$. congensis no crescimento, nutrição mineral e produção. Bragantia, 57:297-312, 1998.

GABELMAN, W.H. \& GERLOFF, G.C. The search for and interpretation of genetic controls that enhance plant growth under deficiency levels of a macronutrient. Plant Soil, 72:335-350, 1983.
GENÚ, P.J.C. Teores de macro e micro nutrientes em folhas de porta-enxertos cítricos (Citrus spp) de pés-francos e em folhas de tangerineira 'Poncã' (Citrus reticulata, Blanco) enxertadas sobre os mesmos porta-enxertos. Piracicaba, Escola Superior de Agricultura "Luiz de Queiroz", 1985. 156p. (Tese de Doutorado)

GERLOFF, G.C. \& GABELMAN W.H. Genetic basis of inorganic plant nutrition. In: LÄUCHLI, A. \& BIELESKI, R.L., eds. Inorganic plant nutrition. Encyclopedia of plant physiology, Berlin, Springer-Verlag, 1983. p.53-480.

JOHNSON, C.M. \& ULRICH, A. Analytical methods for use in plants analyses. Los Angeles, University of California, 1959. p.32-33. (Bulletin, 766)

LI, B.; MCKEAND, S.E. \& ALLEN, H.L. Genetic variation in nitrogen use efficiency of loblolly pine seedlings. For. Sci., 37:613-626, 1991.

MALAVOLTA, E.; VITTI, G.C. \& OLIVEIRA, S.A. Avaliação do estado nutricional das plantas: Princípios e aplicações. 2.ed. Piracicaba, Potafos, 1997. 319p.

MARSCHNER, H. Mineral nutrition of higher plants. London, Academic Press, 1995. 889p.

MARTINEZ, H.E.P.; NOVAIS, R.F.; SACRAMENTO, L.V.S. \& RODRIGUES L.A. Comportamento de variedades de soja cultivadas sob diferentes níveis de fósforo: II. Translocação do fósforo absorvido e eficiência nutricional. R. Bras. Ci. Solo, 17:239-244, 1993.

MORAES M.V. \& FRANCO, C.M. Método expedito para enxertia em café. Rio de Janeiro, Instituto Brasileiro do Café, 1973. 8p.

MOURA, W.M.; CASALI, V.W.D.; CRUZ, C.D. \& LIMA, P.C. Divergência genética em linhagens de pimentão em relação à eficiência nutricional de fósforo. Pesq. Agropec. Bras. 34:217-224, 1999.

SANDS, R. \& MULLIGAN, D.R. Water and nutrient dynamics and tree growth. For. Ecol. Manag., 30:91-111, 1990.

SIDDIQI, M.Y. \& GLASS, A.D.M. Utilization index: A modified approach to the estimation and comparison of nutrient utilization efficiency in plants. J. Plant Nut., 4:289-302, 1981.

SOUZA, C.A.S.; GUIMARÃES, P.T.G.; FURTINI NETO, A.E. \& NOGUEIRA, F.D. Efeito de doses de zinco via solo em três cultivares de cafeeiro (Coffea arabica L.). Ci. Agrotec., 25:890-899, 2001.

TENNANT, D. A test of a modified line intersect method of estimating root length. J. Appll. Ecol., 63:995-1001, 1975.

WILLSON, K.C. Mineral nutrition and fertilizer needs. In: CLIFFORD, M.M.N \& WILLSON, K.C., eds. Coffee: botany, biochemistry and productions of beans and beverage. London, Croom Helm, 1987. p.135-156. 